\title{
Large-scale biophysically detailed model of somatosensory thalamocortical circuits in NetPyNE
}

\author{
Fernando S. Borges ${ }^{1,2}$, Joao V.S. Moreira ${ }^{1}$, Lavinia M. Takarabe ${ }^{2}$, William W. Lytton ${ }^{1,3}$, \\ Salvador Dura-Bernal ${ }^{1,4}$ \\ ${ }^{1}$ Department of Physiology and Pharmacology, State University of New York Downstate Health \\ Sciences University, Brooklyn, NY, USA \\ ${ }^{2}$ Center for Mathematics, Computation, and Cognition, Federal University of ABC, São Paulo, Brazil \\ ${ }^{3}$ Department of Neurology, Kings County Hospital Center, Brooklyn, NY, USA \\ ${ }^{4}$ Nathan Kline Institute for Psychiatric Research, Orangeburg, NY, USA \\ * Correspondence: \\ Corresponding Author \\ fernandodasilvaborges@gmail.com, salvador.dura-bernal@downstate.edu
}

Keywords: somatosensory cortex, thalamocortical circuits, large-scale model, biophysical, cortical, multiscale

\begin{abstract}
The primary somatosensory cortex (S1) of mammals is critically important in the perception of touch and related sensorimotor behaviors. In 2015 the Blue Brain Project developed a groundbreaking rat S1 microcircuit simulation with over 31,000 neurons with 207 morpho-electrical neuron types, and 37 million synapses, incorporating anatomical and physiological information from a wide range of experimental studies. We have implemented this highly-detailed and complex model S1 model in NetPyNE, using the data available in the Neocortical Microcircuit Collaboration Portal. NetPyNE provides a Python high-level interface to NEURON and allows defining complicated multiscale models using an intuitive declarative standardized language. It also facilitates running parallel simulations, automates the optimization and exploration of parameters using supercomputers, and provides a wide range of built-in analysis functions. This will make the $\mathrm{S} 1$ model more accessible and simpler to scale, modify and extend in order to explore research questions or interconnect to other existing models. Despite some implementation differences, the NetPyNE model closely reproduced the original cell morphologies, electrophysiological responses, spatial distribution for all 207 cell types; and the connectivity properties of the 1941 pathways, including synaptic dynamics and short-term plasticity (STP). The NetPyNE S1 simulations produced reasonable physiological rates and activity patterns across all populations, and, when STP was included, produced a $1 \mathrm{~Hz}$ oscillation comparable to that of the original model. We also extended the model by adding thalamic circuits, including 6 distinct thalamic populations with intrathalamic, thalamocortical and corticothalamic connectivity derived from experimental data. Overall, our work provides a widely accessible, data-driven and biophysically-detailed model of the somatosensory thalamocortical circuits that can be utilized as a community tool for researchers to study neural dynamics, function and disease.
\end{abstract}


bioRxiv preprint doi: https://doi.org/10.1101/2022.02.03.479029; this version posted February 6, 2022. The copyright holder for this preprint

(which was not certified by peer review) is the author/funder. All rights reserved. No reuse allowed without permission.

Large-scale model of somatosensory thalamocortical circuits

\section{Introduction}

The primary somatosensory cortex (S1) of mammals is critically important in the perception of touch and works closely with other sensory and motor cortical regions in permitting coordinated activity with tasks involving grasp (Petrof, Viaene, and Sherman 2015; Barthas and Kwan 2017). Moreover, the communication of these cortical areas with the thalamus is crucial for maintaining functions, such as sleep and wakefulness, considering that the thalamocortical (TC) circuit is essential for cerebral rhythmic activity (O'Reilly et al. 2021). A greater understanding of S1 cortical circuits will help us gain insights into neural coding and be of assistance in determining how disease states such as schizophrenia, epilepsy and Parkinson's disease lead to sensory deficits or uncoordinated movement (Petrof, Viaene, and Sherman 2015; Vázquez, Salinas, and Romo 2013; Azarfar et al. 2018; Peña-Rangel et al. 2021).

There exists an impressive, highly-detailed model of rat S1 developed by the Blue Brain Project (BBP) (Markram et al. 2015), incorporating anatomical and physiological information from a wide range of experimental studies. This groundbreaking model includes over 31,000 neurons of 55 layer-specific morphological and 207 morpho-electrical neuron subtypes, and 37 million synapses capturing layer- and cell type-specific connectivity patterns and synaptic dynamics. Simulation results matched in vitro and in vivo experimental findings, and has been used over the years to reproduce additional experimental results and generate predictions of the dynamics and function of cortical microcircuits (Michael W. Reimann et al. 2015; Gal et al. 2017; Michael W. Reimann, Horlemann, et al. 2017; Michael W. Reimann, Nolte, et al. 2017; Amsalem et al., n.d.; Hagen et al. 2018). Although the BBP S1 model is state-of-the-art, certain constraints limit its reproducibility and use by the community, as well as its extension or modification to connect to other regions or update model features. The size and complexity of any model of this scope is daunting. Due to its scale and complexity, the original model must be run and analyzed on large High Performance Computing platforms (HPCs), which are not available to many users. Although the model is simulated using NEURON (Carnevale and Hines 2006; Migliore et al. 2006)a widely used platform within the computational neuroscience community, it also requires other custom libraries specifically designed to facilitate this workflow. These libraries are used to build, manage simulations and analyze the model. However, not all of these libraries and workflows are publicly available (Markram et al. 2015), making it somewhat difficult to modify the code, and scale or simplify the model for simulation on smaller computers, overall reducing its accessibility and reproducibility (McDougal, Bulanova, and Lytton 2016).

Here we implemented the original BBP S1 model in NetPyNE (Dura-Bernal et al. 2019) in order to make it more accessible and simpler to scale, modify and extend. NetPyNE is a python package that provides a high-level interface to the NEURON simulator, and allows the definition of complex multiscale models using an intuitive declarative standardized language. NetPyNE translates these specifications into a NEURON model, facilitates running parallel simulations, automates the optimization and exploration of parameters using supercomputers, and provides a wide range of built-in analysis functions. Conversion to NetPyNE also makes it easier to connect to previous models developed within the platform, such as our primary motor cortex model (Dura-Bernal et al., n.d.; Sivagnanam et al. 2020), and models implemented in other tools (e.g. NEST) by exporting to the NeuroML or SONATA standard formats. In prior work, we ported a classic model of generic sensory cortical circuits (Potjans and Diesmann 2014) to our NetPyNE platform (Romaro et al. 2021) in order to make it both more scalable and facilitate modification of cell models and network parameters; the original model used integrate-and-fire neurons and we replaced these with more complex multi-compartment neuron models. 
bioRxiv preprint doi: https://doi.org/10.1101/2022.02.03.479029; this version posted February 6, 2022. The copyright holder for this preprint (which was not certified by peer review) is the author/funder. All rights reserved. No reuse allowed without permission.

Large-scale model of somatosensory thalamocortical circuits

Although we have primarily focused on simplifying the network description, we have also made the model more complex, and more complete, by adding the associated somatosensory thalamic circuits and bidirectional connectivity with cortex to allow interplay of these two highly coordinated areas (Meyer et al. 2010). The deepening of knowledge about the cortico-thalamo-cortical loop (Shepherd and Yamawaki 2021) should contribute to investigations on rhythmic dysfunctions, such as epilepsy and schizophrenia. But in contrast to cortical microcircuitry, few detailed models exist for the thalamus (Hill and Tononi 2005; Iavarone et al. 2019; Izhikevich and Edelman 2008; Murray and Anticevic 2017).

In this study we present a NetPyNE implementation of the BBP S1 model, capturing most of the original single-cell physiology and morphology, synaptic mechanisms, connectivity and basic simulation results. With the addition of detailed thalamic circuits we extend the results to show synchronous activity across cortical and thalamic populations, and open the door to new investigations on corticothalamic dynamics. The model is able to port readily across machines and can utilize a fast, efficient implementation on CPUs and GPUs using coreNEURON. This extension allows the original BBP S1 model to be readily available to be used by the wider community to study a wide range of research questions.

\section{Material and Methods}

\subsection{Individual neuron models}

To port the somatosensory microcircuit model in NetPyNE (Dura-Bernal et al. 2019), we recreated the single neuron models using cell files from the Neocortical Microcircuit Collaboration (NMCP https://bbp.epfl.ch/nmc-portal) (Ramaswamy et al. 2015). The full dataset comprises 207 morpholectric (me) cell types, with 5 examples for each, totaling 1035 cell models, each stored with morphology file, descriptions of ion channels, and a NEURON HOC template to instantiate the cell, which can be imported directly to NetPyNE (Fig 1). Neuron morphologies from the BBP S1 model (specifically, L1_DLAC, L4_DBC, L23_PC and L6_TPC_L4) imported into NetPyNE were visualized using the NetPyNE GUI (Fig 1A).

Benchmark testing validated physiological responses (Fig 1B-E) at 3 current clamp amplitudes $(120 \%, 130 \%$, and $140 \%$ of threshold; only $120 \%$ shown). Slight differences were observed in the cell types with a stochastic version of the $\mathrm{K}^{+}$channel mechanism (StochKv; Fig. 1D) where we used a deterministic version of the channel from OpenSourceBrain (Gleeson et al. 2019; Gleeson et al. 2019b). The StochKv NMODL (.mod) mechanism required additional code outside of NetPyNE in order to update its state, and the inclusion of stochastic variables in each section of the cells significantly increased the simulation time. In order to understand $\mathrm{StochKv}$ effect on cell response, we applied a current clamp $(0.1 \mathrm{nA}, 2 \mathrm{~s})$ to the soma of each of the 1035 cells, and used the Electrophys Feature Extraction Library (eFEL) (eFEL 2015) to compare BBP and NetPyNE mean firing rate (Fig. 1F) and time to first spike (Fig. 1G) for those with and without the StochKv channel. As expected, variability with the StochKv channel in the original model was pronounced. Although present in 54/207 me-types, the StochKv channels are only in 3.63\% of all cells. Within each m-type (morphology) those with StochKv also correspond to a minority of e-types (electrical) types; for example, only $32 \%$ of L4_DBC cells have e-type bIR (with StochKv channels). Given the small proportion of cells with StochKv channels, the NetPyNE mean firing rates per m-type population closely match those of the original BBP model (Fig. 1H). 
bioRxiv preprint doi: https://doi.org/10.1101/2022.02.03.479029; this version posted February 6, 2022. The copyright holder for this preprint

(which was not certified by peer review) is the author/funder. All rights reserved. No reuse allowed without permission.

Large-scale model of somatosensory thalamocortical circuits

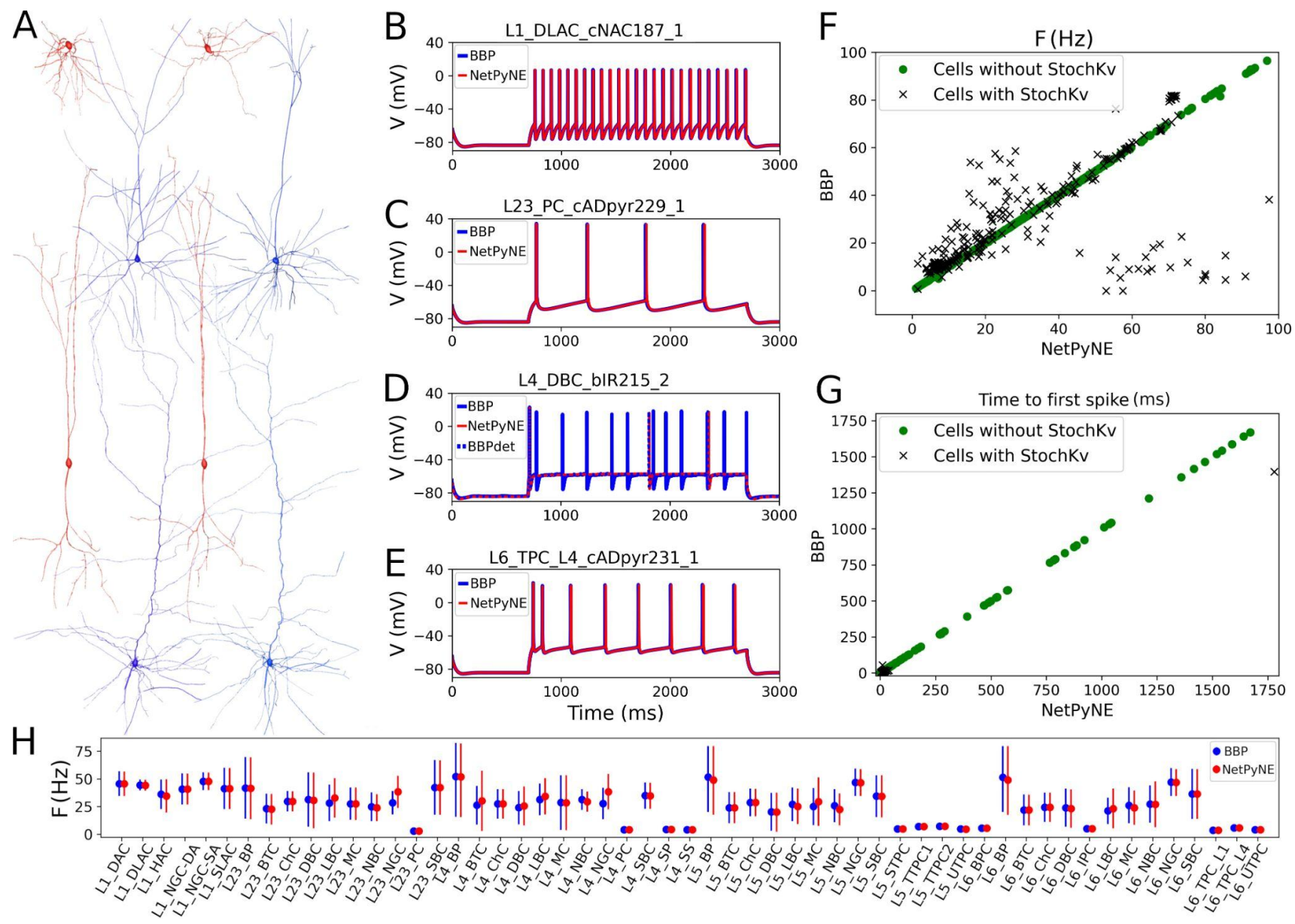

Figure 1. Reproduction and validation of BBP S1 cell types in NetPyNE. (A) 3D reconstructions of 4 pairs of m-type example neurons visualized using the NetPyNE graphical user interface: inhibitory cells L1_DLAC and L4_DBC (red), and excitatory cells L23_PC and L6_TPC_L4 (blue). (B-E) Somatic membrane potential of the neurons in (A) under current clamp with amplitude $120 \%$ of the neuron firing threshold. NetPyNE results (red) compared to the original BBP model results (blue). For L4_DBC_bIR cells (panel D) we used a deterministic version of the BBP stochastic potassium channel (StochKv) resulting in divergent results; using same deterministic channel in BBP (BBPdet, blue dotted line) restores the match to NetPyNE results. (F,G) Comparison of BBP and NetPyNE firing rate and time to first spike in response to current-clamp with amplitude $0.1 \mathrm{nA}$ during 2 seconds for each cell type. Only some cell types with the StochKv show differences. (H) Comparison BBP and NetPyNE mean firing rate for all m-type populations. Due to the small number of cells with StochKv (3.6\%), NetPyNE population firing rates closely match those of BBP.

\subsection{Distribution and connectivity of cortical populations}

Rather than instantiating connectivity from a list of individual synapses based on anatomical overlap of neuronal arbors (M. W. Reimann et al. 2015), we created our S1 port using probability rules for both neuron distribution and connections. The network consisted of 31,346 cells in a cylindrical volume $2082 \mu \mathrm{m}$ height and radius of $210 \mu \mathrm{m}$ as in the original model (Fig 2). Each population was randomly distributed within its specific layer (L1, L2/3, L4, L5, or L6). The number of cells in each one of 207 me-types was taken from the NMCP (Ramaswamy et al. 2015) the minicolumn data available was not used to distribute cells. A 2D representation of the cell distribution within the cylindrical volume is shown in Fig. 2A, with layer thicknesses (in $\mu \mathrm{m}$ ) for L1, L23, L4, L5, and L6 set to $165,502,190,525$, and 700, respectively. We used the S1 connectome (Gal et al. 2017) from NMCP, following the approach in (Michael W. Reimann, Horlemann, et al. 2017): 7 stochastic 
bioRxiv preprint doi: https://doi.org/10.1101/2022.02.03.479029; this version posted February 6, 2022. The copyright holder for this preprint (which was not certified by peer review) is the author/funder. All rights reserved. No reuse allowed without permission.

Large-scale model of somatosensory thalamocortical circuits

instances of a model microcircuit based on averaged measurements of neuron densities were used to calculate distance-dependent probabilities of connection. In each microcircuit instance, we calculated the connection probability for each pair of neurons based on the 2D somatic distance (horizontal XZ-plane) for each of the 1941 pathways. To estimate the distance dependent probability, we calculated the probability in evenly spaced intervals starting at $25 \pm 25 \mu \mathrm{m}$, in $50 \mu \mathrm{m}$ intervals, up to $375 \pm 25 \mu \mathrm{m}$. Next, we calculated the mean probability across the 7 microcircuits in evenly spaced intervals and used the mean values to fit the connection probability rules. We evaluated multiple functions for each pathway and selected the one that provided the best fit to the data. Figure $2 \mathrm{~B}-\mathrm{D}$ shows how this approach was used to calculate the connection probability of 3 example projections: data from the 7 microcircuit instances (mcs; cyan circles), is averaged across microcircuits (green diamonds) and fitted to either a single exponential (Fig. 2B, red line); an exponential with a linear saturation rule (Fig. 2C), or a single gaussian (Fig. 2D, dashed line). Because the original S1 model shows high variability in the number of synapses per connection, we calculated the mean values for each pathway and used it as a parameter in our model. The result is a representative reconstruction of the S1 column connectivity in NetPyNE, with approximately 27.6 million excitatory synapses and 9.6 million inhibitory synapses.

Using the fitted rules, we reconstructed an entire S1 column in NetPyNE and compared the two versions using the mean number of connections. To avoid overfitting, we generated 7 different instances using different connectivity seeds for both the NetPyNE and BBP models. The number of connections was similar in both models for each of the 1941 pathways (Fig. 2E) and for each of the four projection types (p-type) (EE, EI, IE, II) (Fig. 2F). 
bioRxiv preprint doi: https://doi.org/10.1101/2022.02.03.479029; this version posted February 6, 2022. The copyright holder for this preprint

(which was not certified by peer review) is the author/funder. All rights reserved. No reuse allowed without permission.

Large-scale model of somatosensory thalamocortical circuits
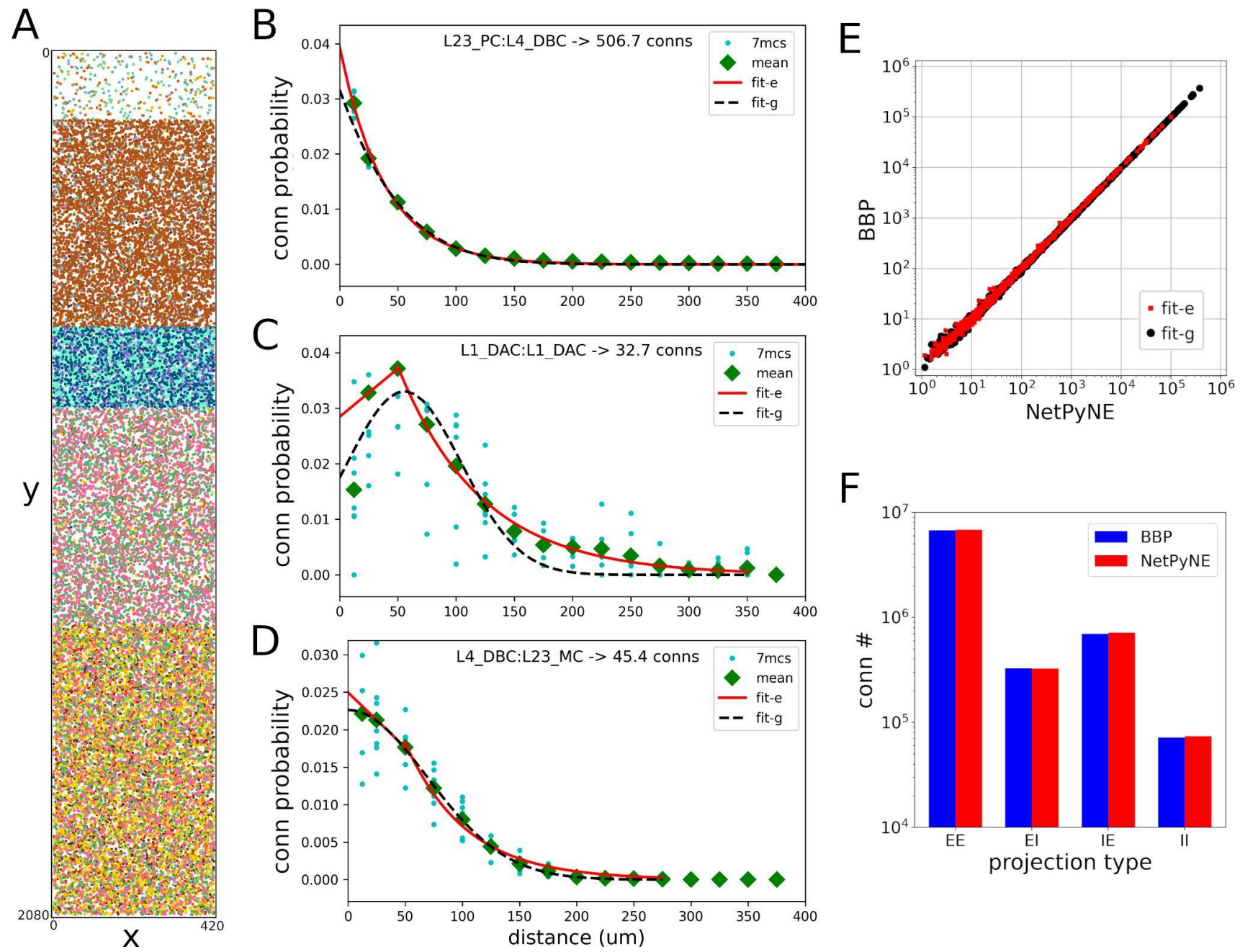

Figure 2. Reproduction and validation of BBP S1 neuron distribution and connectivity in NetPyNE (A) 2D representation of the location of 31,346 cells in a cylinder with 2082 um height and 210 um radius, with each subtype (different colors) randomly distributed within its layer (L1, L2/3, L4, L5, or L6). (B-D) Probability of connection as a function of neuron pairwise 2D distance for three example pathways, each with a different best fit function: a single exponential (B, red line), exponential with a linear saturation rule $(\mathrm{C}$, red line), and single gaussian fit (D, black dashed line). Cyan circles represent data from 7 microcircuit instances, and green diamonds represent the mean across the 7 instances. (E-F) Comparison of the number of connections between NetPyNE and BBP for each of the 1941 pathways (E) and 4 projection types (p-types) (F).

\subsection{Synaptic physiology}

The original BBP S1 model included detailed synaptic properties (conductances, post-synaptic potentials, latencies, rise and decay times, failures, release probabilities, etc) recapitulating published experimental data. Short-term dynamics were used to classify synapses into the following types (s-types): inhibitory facilitating (I1), inhibitory depressing (I2), inhibitory pseudo-linear (I3), excitatory facilitating (E1), excitatory depressing (E2), and excitatory pseudo-linear (E3). A set of rules were then derived from experimental data to assign an s-type to each broad class of connections. Based on the NMCP data, there were 29 classes of connections as determined by the combination of pre- and post-synaptic me-types. The synaptic properties, s-type and p-type for each class of connections are summarized in Table 1. Synaptic properties included kinetic parameters: 
bioRxiv preprint doi: https://doi.org/10.1101/2022.02.03.479029; this version posted February 6, 2022. The copyright holder for this preprint (which was not certified by peer review) is the author/funder. All rights reserved. No reuse allowed without permission.

Large-scale model of somatosensory thalamocortical circuits

\begin{tabular}{|c|c|c|c|c|c|c|c|c|c|}
\hline \# & $\begin{array}{c}\text { BBP } \\
\text { id }\end{array}$ & s-type & p-type & $\mathrm{g}_{\text {syn }}(\mathrm{nS})$ & $\tau_{\text {decay }}(\mathbf{m s})$ & $\mathbf{U}$ & D (ms) & $\mathbf{F}(\mathrm{ms})$ & pre- and post-syn cell type rules \\
\hline $\mathbf{0}$ & 0 & $\mathrm{I} 1$ & II & $0.83 \pm 0.55$ & $10.40 \pm 6.10$ & $0.16 \pm 0.100$ & $45 \pm 21$ & $376 \pm 253$ & L6:L6_(DBC-LBC-NBC-SBC) \\
\hline 1 & 3 & I1 & IE & $1 \pm 0.61$ & $10.40 \pm 6.10$ & $6 \pm 0.100$ & $45 \pm 21$ & $6 \pm 253$ & SBC_cAC:Exc or L6_(NBC-LBC):L6_BPC \\
\hline 2 & 13 & $\mathrm{I} 1$ & IE & $0.75 \pm 0.32$ & $10.40 \pm 6.10$ & $0.41 \pm 0.212$ & $162 \pm 69$ & $690 \pm 5$ & L6_MC:L6_IPC \\
\hline 3 & 1 & $\mathrm{I} 2$ & II & $0.83 \pm 0.55$ & $8.30 \pm 2.20$ & $0.25 \pm 0.130$ & $706 \pm 405$ & $21 \pm 9$ & :Excitatory or Inhibitory:Inhibitory \\
\hline 4 & 4 & I2 & IE & $0.91 \pm 0.61$ & $8.30 \pm 2.20$ & $0.25 \pm 0.130$ & $706 \pm 405$ & $21 \pm 9$ & SBC_dNAC:Excitatory \\
\hline 5 & 8 & I2 & IE & $0.75 \pm 0.32$ & $8.30 \pm 2.20$ & $25 \pm 0.130$ & $706 \pm 405$ & $21 \pm 9$ & BTC_DBC-B \\
\hline 6 & 9 & I2 & IE & $0.75 \pm 0.32$ & $8.30 \pm 2.20$ & $0.30 \pm 0.080$ & $1250 \pm 520$ & $2 \pm 4$ & MC:Excitatory \\
\hline 7 & 10 & $\mathrm{I} 2$ & IE & $0.91 \pm 0.61$ & $8.30 \pm 2.20$ & $0.14 \pm 0.050$ & $875 \pm 285$ & $22 \pm 5$ & $\begin{array}{l}\text { LBC-NBC_(bAC cAC bNAC } \\
\text { dNAC):Excitatory }\end{array}$ \\
\hline 8 & 12 & I2 & IE & $2.97 \pm 0.95$ & $8.30 \pm 2.20$ & $0.25 \pm 0.130$ & $706 \pm 405$ & $21 \pm 9$ & Chc:Excitatory \\
\hline 9 & 5 & $\mathrm{I} 3$ & IE & $0.91 \pm 0.61$ & $6.44 \pm 1.70$ & $0.32 \pm 0.140$ & $144 \pm 80$ & $62 \pm 31$ & $\begin{array}{l}\text { SBC_bNAC or LBC-NBC_( } \\
\text { dSTUT cSTUT bSTUT):Exc }\end{array}$ \\
\hline 10 & 11 & I3 & IE & $0.83 \pm 0.55$ & $36.55 \pm 0.71$ & $0.25 \pm 0.130$ & $706 \pm 405$ & $21 \pm 9$ & NGC:Excitatory \\
\hline 11 & 114 & 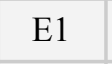 & EI & 28 & $1.74 \pm 0.18$ & $0.02 \pm 0.001$ & $194 \pm 10$ & $507 \pm 20$ & Exc:(BP_cAC DBC_cAC BTC_cAC) \\
\hline 12 & 115 & F1 & EI & $0.72 \pm 0.50$ & $1.74 \pm 0.18$ & $0.02 \pm 0.001$ & $194 \pm 10$ & $507 \pm 20$ & $\begin{array}{c}\text { Exc:(NBC-LBC)_(cAC cIR bAC bIR } \\
\text { cNAC) }\end{array}$ \\
\hline 13 & 132 & E1 & EI & $0.72 \pm 0.50$ & $1.74 \pm 0.18$ & $0.01 \pm 0.001$ & $242 \pm 15$ & $563 \pm 32$ & L6_TPC_L:L6_(DBC-LBC-NBC-SBC \\
\hline 14 & 133 & $\mathrm{E}$ & 1 & $0.11 \pm 0.08$ & $1.14 \pm 0.10$ & $09 \pm 0.120$ & $138 \pm 211$ & $670 \pm 830$ & Excita \\
\hline 15 & 116 & 2 & FF & $0.72 \pm 0.50$ & $1.74 \pm 0.18$ & $0.50 \pm 0.020$ & $671 \pm 17$ & $17+5$ & 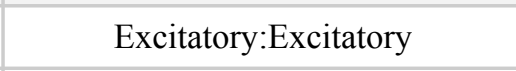 \\
\hline 16 & 117 & E2 & EI & $0.43 \pm 0.28$ & $1.74 \pm 0.18$ & $0.50 \pm 0.020$ & $671 \pm 17$ & $17 \pm 5$ & $\begin{array}{c}\text { Excitatory:[L1-BP_(cNAC } \\
\text { bNAC)-DBC_bAC-BTC_(bAC cNAC bIR)] }\end{array}$ \\
\hline 17 & 118 & E2 & EI & $0.72 \pm 0.50$ & 8 & $0.50 \pm$ & $671 \pm 17$ & $17 \pm 5$ & Excit \\
\hline 18 & 119 & E2 & $\mathrm{EE}$ & $0.68 \pm 0.46$ & $1.74 \pm 0.18$ & $0.46 \pm 0.260$ & $671 \pm 17$ & $17 \pm 5$ & L23_PC:L23_PC \\
\hline 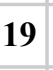 & 120 & E2 & $\mathrm{EE}$ & 0 & 8 & 9 & 7 & 5 & Excitatory:L4_E \\
\hline 20 & 121 & E2 & $\mathrm{EE}$ & $0.19 \pm 0.12$ & $1.74 \pm 0.18$ & $0.79 \pm 0.040$ & $671 \pm 17$ & $17 \pm 5$ & L4_SS:L23_PC \\
\hline 21 & 122 & E2 & EE & $0.80 \pm 0.53$ & $1.74 \pm 0.18$ & $0.39 \pm 0.030$ & $671 \pm 17$ & $1 / \pm 0$ & L5_STPC:L5_STPC \\
\hline 22 & 123 & D & D & $1.50 \pm$ & $1.74 \pm 0$ & 20 & 0 & 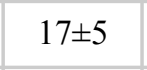 & 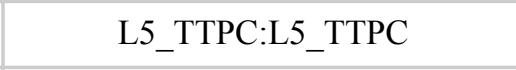 \\
\hline 23 & 127 & E2 & $\mathrm{EE}$ & $0.80 \pm 0.53$ & $1.74 \pm 0.18$ & $0.39 \pm 0.134$ & $780 \pm 54$ & $51 \pm 36$ & L6_IPC:L6_IPC \\
\hline 24 & 131 & $\mathrm{~L} 2$ & EI & $0.72 \pm 0.50$ & $1.74 \pm 0.18$ & $0.58 \pm 0.070$ & $240 \pm 43$ & $71 \pm 47$ & L6_IPC:L6_(DBC-LBC-NBC-SBC) \\
\hline 25 & 134 & E2 & EI & $0.72 \pm 0.50$ & $1.74 \pm 0.18$ & $0.72 \pm 0.065$ & $227 \pm 38$ & $14 \pm 12$ & $\begin{array}{c}\text { Exc:(NBC-LBC)_(bSTUT dNAC } \\
\text { bNAC cSTUT) }\end{array}$ \\
\hline 26 & 126 & E3 & $\mathrm{EE}$ & $0.80 \pm 0.53$ & $1.74 \pm 0.18$ & $0.21 \pm 0.032$ & $460 \pm 53$ & $230 \pm 69$ & L6_TPC_L:L6_TPC_L \\
\hline 27 & 128 & E3 & $\mathrm{EE}$ & $0.80 \pm 0.53$ & $1.74 \pm 0.18$ & $0.27 \pm 0.033$ & $559 \pm 238$ & $200 \pm 92$ & L6_IPC:L6_BPC \\
\hline 28 & 129 & E3 & EE & $0.80 \pm 0.53$ & $1.74 \pm 0.18$ & $0.22 \pm 0.053$ & $535 \pm 134$ & $116 \pm 81$ & L6_IPC:L6_TPC_L \\
\hline
\end{tabular}

Table 1. Synaptic properties, s-type, p-type and rules for each class of connections implemented in NetPyNE. s-type: type of short-term dynamics; $p$-type: type of projection; $\mathrm{g}_{\text {syn }}$ : peak conductance $(\mathrm{ms})$; $\tau_{\text {decay }}$ : decay time (ms); U: neurotransmitter release probability; D: time constant for recovery from depression (ms); F: time constant for recovery from facilitation $(F)$. Values indicate mean \pm standard deviation. 
bioRxiv preprint doi: https://doi.org/10.1101/2022.02.03.479029; this version posted February 6, 2022. The copyright holder for this preprint (which was not certified by peer review) is the author/funder. All rights reserved. No reuse allowed without permission.

Large-scale model of somatosensory thalamocortical circuits

peak conductance $\left(\mathrm{g}_{\text {syn }}\right)$ and decay time $\left(\tau_{\text {decay }}\right)$; and dynamic parameters: neurotransmitter release probability (U), time constant for recovery from depression (D; in $\mathrm{ms}$ ) and time constant for recovery from facilitation ( $\mathrm{F}$; in $\mathrm{ms}$ ). We also included a compact description of the rules to determine what connections belong to each class, based on the pre- and postsynaptic cell types. For clarity, we rearranged the classes of connections by s-types in the sequence I1, I2, I3, E1, E2, and E3 (from 0 to 28), and included the original BBP class label for reference. The parameters D and F correspond to the synapses with short term plasticity (STP), which could be optionally added to recurrent S1 connections, and connections from thalamus to S1.

The s-types for each class of connections and for each of the 1941 pathways are color-coded and illustrated in Fig. 4A. Since pathways depend on m-types but connection classes depend on me-types (each m-type includes multiple me-types), it is possible to have multiple s-types for the same pathway, in those cases we simply labeled it as either I2 or E2. To implement the dynamics of each s-type in NetPyNE we used a deterministic version of the dual-exponential synaptic model (Hennig 2013; Fuhrmann et al. 2002). Example simulations of the post-synaptic potentials for the different s-types are shown in Fig 3B. For each example, we ran 20 simulations with 5 different post-synaptic cells of the same me-type and 4 random synaptic distributions. Pre- and post-synaptic neurons of specific me-types were selected to illustrate each of the six s-types (see Fig. 3 caption). 
bioRxiv preprint doi: https://doi.org/10.1101/2022.02.03.479029; this version posted February 6, 2022. The copyright holder for this preprint (which was not certified by peer review) is the author/funder. All rights reserved. No reuse allowed without permission.

Large-scale model of somatosensory thalamocortical circuits
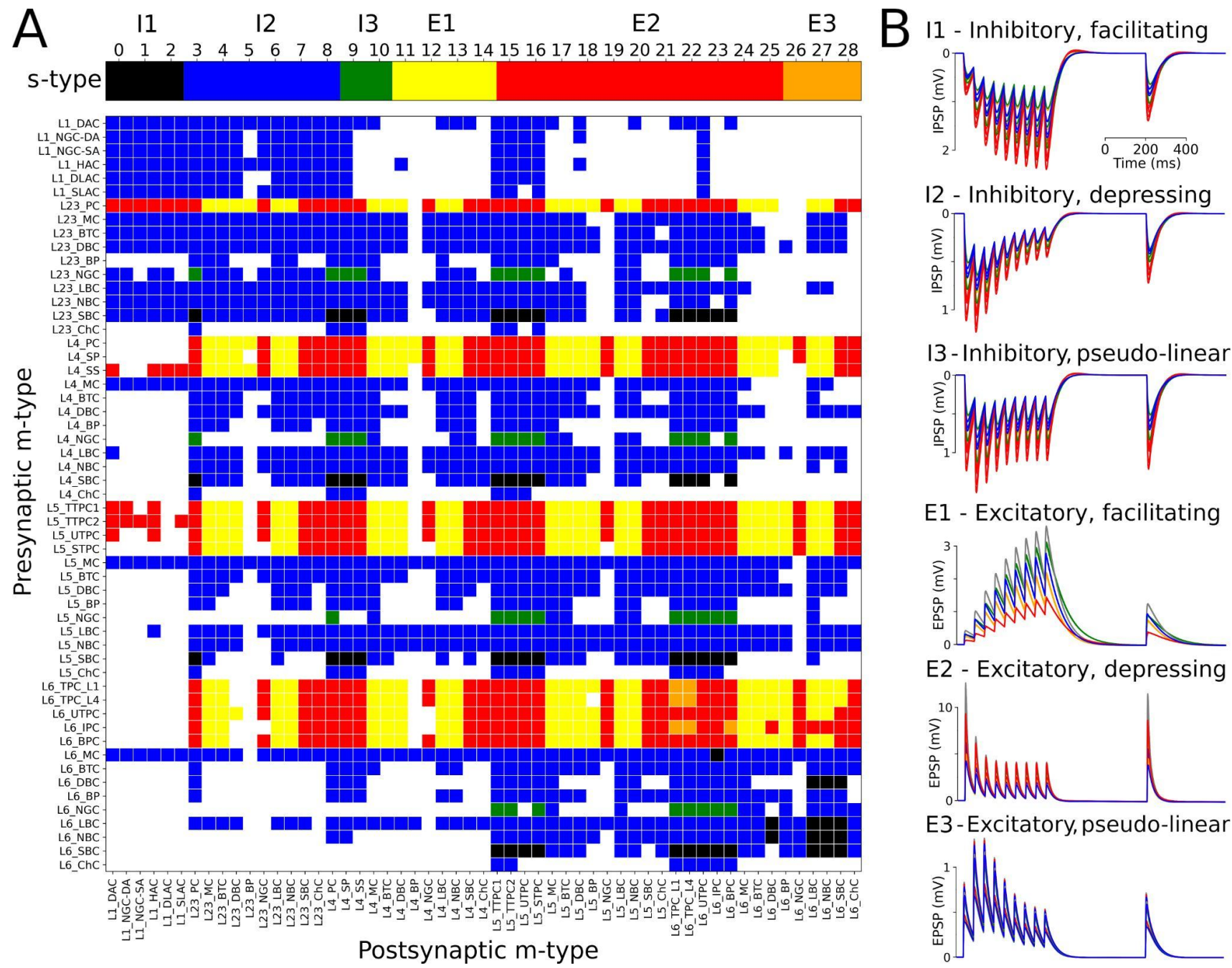

13 - Inhibitory, pseudo-linear

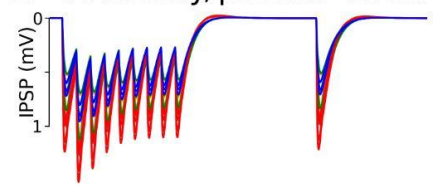

E1 - Excitatory, facilitating

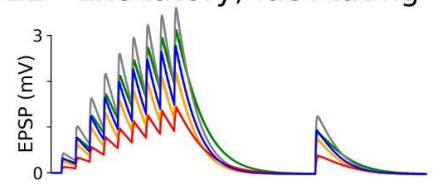

E2 - Excitatory, depressing

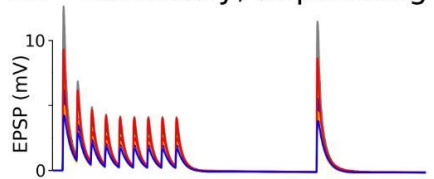

E3-Excitatory, pseudo-linear

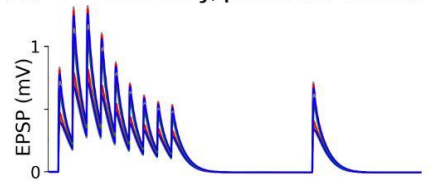

Figure 3. Matrix of s-types for each of the 1941 pathways and simulated PSPs exemples for each s-type in NetPyNE. (A) Color-coded s-types for each class of connections (top) and for each of the 1941 pathways (bottom). Note that for pathways with multiple s-types only either I2 or E2 is shown. (B) Example simulations of post-synaptic potentials to illustrate each of the six s-types. Each example shows the results of 20 simulations with 5 different post-synaptic cells (different colors) of the same me-type, and 4 random synaptic distributions. Inhibitory s-types were simulated using pathway L23_SBC:L23_PC, which included different s-types depending on pre-synaptic e-type: I1 for e-type cAC, I2 for e-type dNAC, and I3 for e-type bNAC (as shown in Table 1). Excitatory s-types E1 and E2 were simulated using pathway L23_PC:L23_LBC, e-types cAC and dNAC, respectively; and s-type E3 was simulated using pathway L6_TPC_L4:L6_TPC_L4.

\subsection{Extending the model to include thalamic populations and connectivity}

We extended the model to include somatosensory thalamic populations with cell type-specific dynamics, intra-thalamic connectivity and bidirectional projections with cortex. In the original model, thalamic inputs were modeled as spike generators that only provided feedforward inputs to S1. Our somatosensory thalamus model is composed of the excitatory ventral posterolateral (VPL), ventral posteromedial (VPM) and the posteromedial (POm) nuclei, and the inhibitory reticular nucleus (RTN). We used single compartment cell models with dynamics tuned to reproduce previous studies on the interaction between the thalamic relay and reticular cells (Destexhe et al. 1996), but adjusted to work in large-scale networks (Moreira et al. 2021). The thalamic circuit architecture consisted of six stacked populations as a rough approximation of the thalamic anatomical layout (Fig 
bioRxiv preprint doi: https://doi.org/10.1101/2022.02.03.479029; this version posted February 6, 2022. The copyright holder for this preprint (which was not certified by peer review) is the author/funder. All rights reserved. No reuse allowed without permission.

Large-scale model of somatosensory thalamocortical circuits

4A). The top three were inhibitory populations comprising the outer, middle and inner sectors of the RTN, and spanning a height of $78 \mu \mathrm{m}, 78 \mu \mathrm{m}$ and $156 \mu \mathrm{m}$, respectively. Below these were the three excitatory populations, VPL, VPM and POm, with heights of $156 \mu \mathrm{m}, 156 \mu \mathrm{m}$ and $312 \mu \mathrm{m}$, respectively. The horizontal dimensions (X-Z plane) for all populations were $420 \mu \mathrm{m}$ x $420 \mu \mathrm{m}$. Cells were randomly distributed across each nuclei with the number of cells in each population based on cellular density obtained from the Cell Atlas for the Mouse Brain (https://bbp.epfl.ch/nexus/cell-atlas/) (Erö et al. 2018). Although POm was larger than VPL and VPM, we reduced its cell density by $50 \%$, resulting in approximately the same population size. This lower density accounts for the proportion of coexisting, but functionally-isolated, M1-projecting thalamocortical cells present in POm with no projections to S1 (Guo et al. 2020).

The intrathalamic connectivity was based on data of axonal and dendritic footprints for each nucleus. The VPL and VPM are considered first-order nuclei (FO), which means they receive afferent information from peripheral sensory organs (not modeled here) and are interconnected with cortex (Ma 1991; Sugitani et al. 1990; Łuczyńska et al. 2003) and RTN (Lam and Sherman 2011) in a topological fashion. On the other hand, POm is considered a higher-order (HO) nucleus, so input arrives mainly from the cortex, in this case, from S1 L5 and L6 (O'Reilly et al. 2021; Ohno et al. 2012). The connectivity pattern of HO nuclei has not been properly characterized, but literature reports a decreased level of organization of HO nuclei inputs to RTN (Lam and Sherman 2011), as it sends projections to $\mathrm{S} 1$.

We therefore adopted two connectivity strategies. In the first, neurons from FO nuclei projected with a column-like topological organization. We implemented this by combining a probability of connection that decreased exponentially with the horizontal distance between the pre- and post-synaptic cells with a decay constant proportional to the footprint radius, and which was truncated to 0 outside of the footprint radius. The following footprint diameters were derived from experimental data (or estimated in the case of no literature reports) for the different axonal footprints of each thalamic projection: RTN $\rightarrow$ RTN: $264.63 \mu \mathrm{m}$ (Lam, Nelson, and Sherman 2006); RTN $\rightarrow$ VPL and RTN $\rightarrow$ VPM: $64.33 \mu \mathrm{m}$ (Lam and Sherman 2007); VPL $\rightarrow$ RTN: $97.67 \mu \mathrm{m}$; and VPM $\rightarrow$ RTN: $103.57 \mu \mathrm{m}$ (Lam and Sherman 2011). The second strategy was a divergence rule, with the number of projections from and to $\mathrm{HO}$ nuclei having a fixed value and being distributed without spatial constraints. This divergence value was adjusted so that the $\mathrm{HO}$ dynamics resembled that of the FO nuclei. This allowed us to replicate a column-like topological organization in FO nuclei using single-compartment cells (Lam and Sherman 2007), and distribute the HO connections to behave in a functionally similar fashion (Fig. 4B).

All excitatory thalamic nuclei were indirectly interconnected through their RTN projections, which was divided into three sectors, in line with reports of preferred innervation zones by each of the thalamic nuclei (Lam and Sherman 2011). Synapses within RTN were mediated by $\mathrm{GABA}_{\mathrm{A}}$, from RTN to the excitatory nuclei by a combination of GABAa and GABAb with equal weight, and by AMPA from the excitatory nuclei to RTN and cortex (Destexhe et al. 1996). The probability and weight of connections were the targets of parameter optimization. The matrix with the convergence of intra-thalamic connections is shown in Fig. 4E.

Feedback corticothalamic connectivity originated from S1 m-types L5_TTPC2 and L6_TPC_L4 (O'Reilly et al. 2021). Similar to the topological rules described above, we implemented connectivity with convergence of 30 (i.e. number of pre-synaptic cells projecting to each post-synaptic cell), but only if the horizontal distance between the pre- and post-synaptic neurons was lower than $50.0 \mu \mathrm{m}$ (Fig. 4). 
bioRxiv preprint doi: https://doi.org/10.1101/2022.02.03.479029; this version posted February 6, 2022. The copyright holder for this preprint (which was not certified by peer review) is the author/funder. All rights reserved. No reuse allowed without permission.

Large-scale model of somatosensory thalamocortical circuits

Thalamocortical connectivity from VPL, POm and VPM to S1 was implemented using convergence values estimated from previous studies (Meyer et al. 2010) (Fig. 4C). Convergence values for each of the $55 \mathrm{~m}$-types were calculated based on the weighted average of the populations in each layer. The convergence values for inhibitory populations were multiplied by a scaling factor derived from the original mode $(\sim 0.595)$. This thalamic convergence factor for inhibitory cells was estimated by dividing the IE ratio of VPM thalamic innervation $(83 / 775=0.107)$ by the average IE population ratio $(4,779 / 26,567=0.18)$. The resulting $\mathrm{S} 1$ column received approximately $4.95 \mathrm{M}$ synapses from VPM, 4.95M from VPL, and 3.1M from POm. This is consistent with values that can be derived from experimental studies (Meyer et al. 2010), with 4.27M synapses from VPM and, and 2.66M from POm.
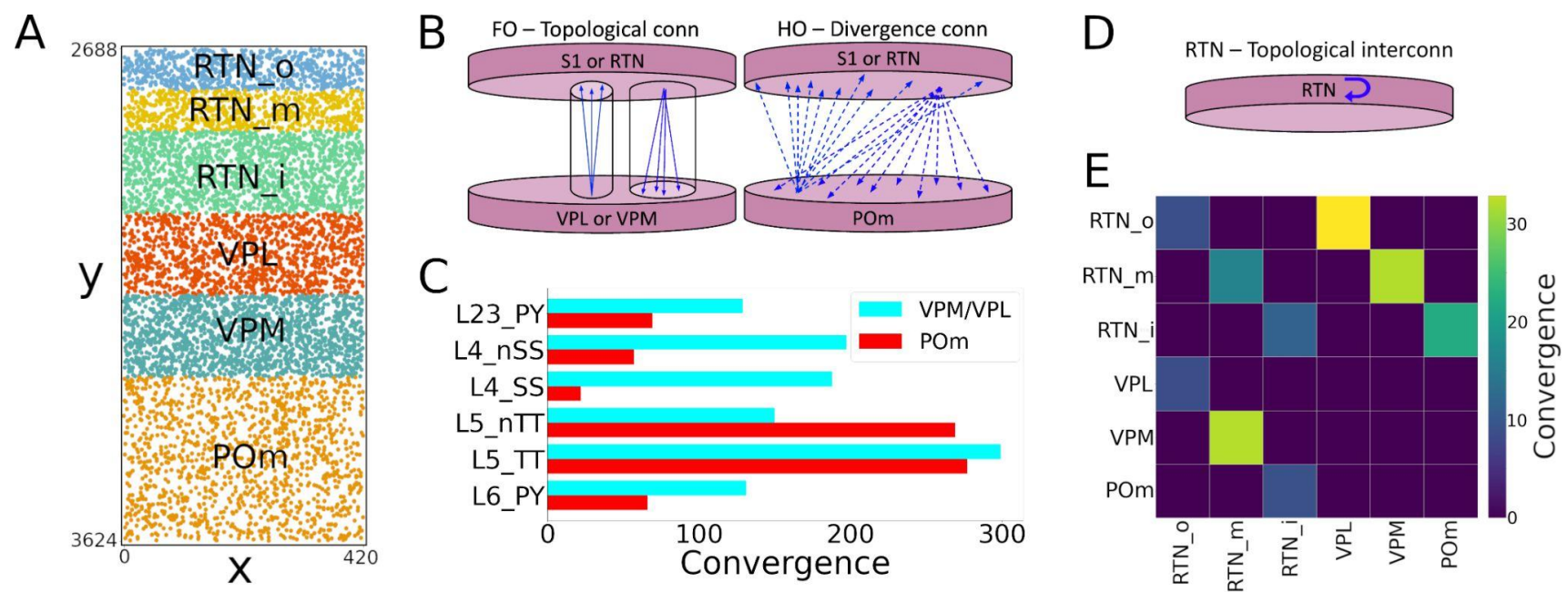

Figure 4. NetPyNE model of somatosensory thalamic populations and connectivity extending the original S1 model. (A) Distribution of neurons across the six different thalamic populations, roughly mimicking the thalamus anatomy. $\mathrm{X}$ and $\mathrm{Y}$ axes in $\mu \mathrm{m}$. (B) Schematic of bidirectional connectivity between thalamic regions and cortex. Bidirectional connections between S1/RTN and first order (FO) regions VPL and VPM are topological, whereas those with high order (HO) region POm are non-topological and implemented using divergence rules. (C) Convergence connectivity between thalamic regions and S1 (D) RTN cells have sector-specific topological connectivity (E) Convergence connectivity matrix across all thalamic populations.

\subsection{Background inputs}

Each cell in the S1 circuit received 10 synaptic inputs from Poisson-distributed spike generators (NetStims) to represent the global effect of spontaneous synapses, background, and other noise sources from non-modeled brain regions projecting to S1. These stimuli were randomly distributed randomly across all sections. The quantal synaptic conductance was calculated based on the average quantal conductance for excitatory and inhibitory synapses. We tuned the excitatory and inhibitory stimuli rates using grid search parameter exploration to obtain average excitatory firing rates of $\sim 1 \mathrm{~Hz}$ and physiological firing rates for all S1 populations.

\subsection{Model building and simulations}

We used the NetPyNE modeling tool (Dura-Bernal et al. 2019) to build, manage simulations, and analyze results of the S1 and thalamic circuit model. NetPyNE employs NEURON (Lytton et al. 2016; Carnevale and Hines 2006; Migliore et al. 2006) as backend simulation engine, with either the standard or coreNEURON libraries (Kumbhar et al. 2019). The high-level Python-based declarative language provided by NetPyNE facilitated the development of this highly complex and extensive 
bioRxiv preprint doi: https://doi.org/10.1101/2022.02.03.479029; this version posted February 6, 2022. The copyright holder for this preprint (which was not certified by peer review) is the author/funder. All rights reserved. No reuse allowed without permission.

Large-scale model of somatosensory thalamocortical circuits

circuit model. This language enabled us to easily import existing morphological and biophysical parameters of different cell types, and define complex connectivity and stimulation rules. We used NetPyNE to explore and optimize the model parameters through automated submission and managing of simulations on supercomputers. We also employed NetPyNE's built-in analysis functions to plot 2D representations of cell locations, connectivity matrices, voltage traces, raster plots and firing rate statistics. NetPyNE can also be used to export the model into the NeuroML (Gleeson et al. 2010) and SONATA (Dai et al. 2020) standard formats.

\section{$3 \quad$ Results}

\subsection{Reproduction of cell morphologies, physiological responses, spatial distribution and connectivity}

Cells imported into NePyNE using the files from The Neocortical Microcircuit Collaboration NMCP (Ramaswamy et al. 2015), reproduced the morphological and electrophysiological characteristics of the original model (Fig 1): RMP and time to the first spike after a current clamp stimulation are fitted for all the 1035 cell types. Firing dynamic differences were observed in cells with the stochastic K channel (StochKv), but given their low proportion $(3.63 \%)$, the average firing rates of all m-type populations closely matched those in the original model (Fig. 1H).

We were able to recreate the general characteristics across the 7 BBP S1 microcircuit instances: the 31,346 cells distributed randomly by layer, and probabilistic connections are generated for each of the 1941 pathways (Fig. 2). This network parameterization allowed us to rescale the microcolumn and generate different instances by changing random number generator seed. Our probabilistic rules best reproduced the original number of connections using a Gaussian fit in most projection pathways (1303 of 1941) and an exponential fit plus a linear saturation in the remaining 638 cases (Fig. 2).

\subsection{Extension to include detailed thalamic circuits}

We extended the model to include the somatosensory thalamic populations with projections to S1: RTN, POm, VPL, and VPM. The number of thalamic cells was adapted to fit a cylindrical column with the same radius as the $\mathrm{S} 1$ column. This facilitated the inclusion of topological connectivity rules between the two regions. We reproduced the firing dynamics of the different thalamic cell types using a single compartment neuron model (Moreira et al. 2021). The connections from TC cells to S1 were based on convergence rules derived from experimental data (Meyer et al. 2010), and synaptic physiological mechanisms were generalized from the BBP VPM projections to S1 layers 4 and 5 (Markram et al. 2015). Feedback connections originated from S1 cell types L5_TTPC2 and L6_TPC_L4 and targeted VPL and VPM following a topological organization, and POm in a following a non-topological broader distribution (Fig. 4). The parameters of the thalamic circuit were adjusted to reproduce a stable self-sustained activity with rhythmic bursting and spindle oscillations, as well as a shift in dynamics following localized excitatory input in the relay cells (Moreira et al. 2021).

\subsection{Circuit response to background inputs (spontaneous activity)}

When driven with background inputs, in the absence of thalamic inputs, the S1 model generated spontaneous activity with all population firing within physiological rates (Fig 5). To achieve this, excitatory and inhibitory background inputs were tuned via grid search parameter optimization (see Methods). Simulations were ran using NetPyNE and NEURON on a Google Cloud virtual machine with 40 cores. We compared simulation results using the standard NEURON simulation engine vs 
bioRxiv preprint doi: https://doi.org/10.1101/2022.02.03.479029; this version posted February 6, 2022. The copyright holder for this preprint (which was not certified by peer review) is the author/funder. All rights reserved. No reuse allowed without permission.

Large-scale model of somatosensory thalamocortical circuits

CoreNEURON, a state-of-the-art version optimized for large scale parallel simulations on both CPUs and GPUs (Kumbhar et al. 2019). Fig. 5 shows the S1 spontaneous activity results, including a spiking raster plot of all 31,346 cortical cells, examples of voltage traces for each of the 207 me-type population, and the average firing rates for each of the 55 m-type populations for NEURON vs CoreNEURON. Both simulation engines produced very similar firing rates for each population, with excitatory and L1-L3 inhibitory cells showing overall lower firing rates than L4-L6 inhibitory cells. The overall average firing rate was $0.95 \mathrm{~Hz}$ in both cases (NEURON: 59,779 spikes; CoreNEURON: 59,749 spikes). This demonstrates the consistency of results obtained from both simulation engines, making CoreNEURON a viable alternative to study the S1 network. CoreNEURON was 2.4x faster to create the network and $2.2 x$ faster to run the simulation (Fig 5D).
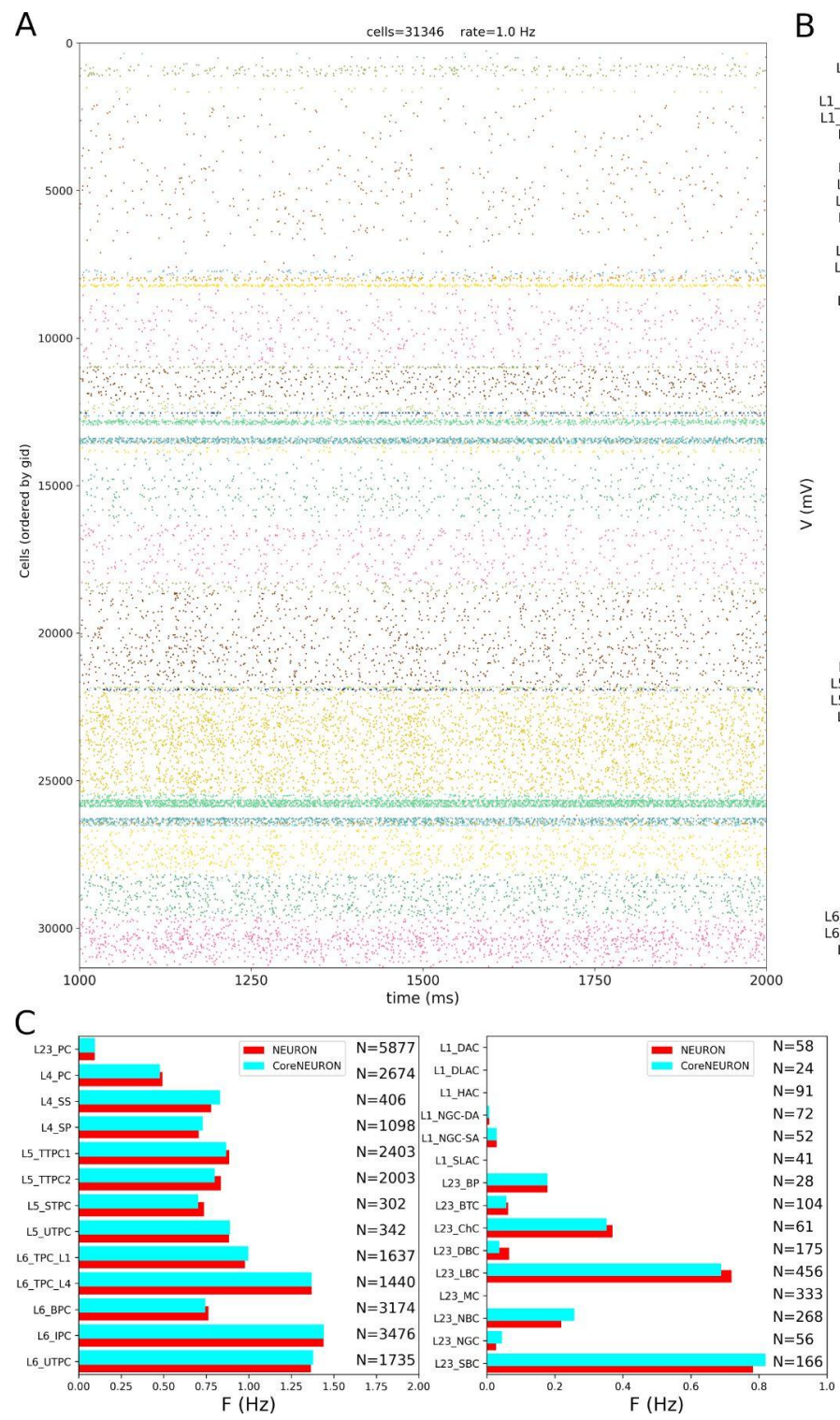

B
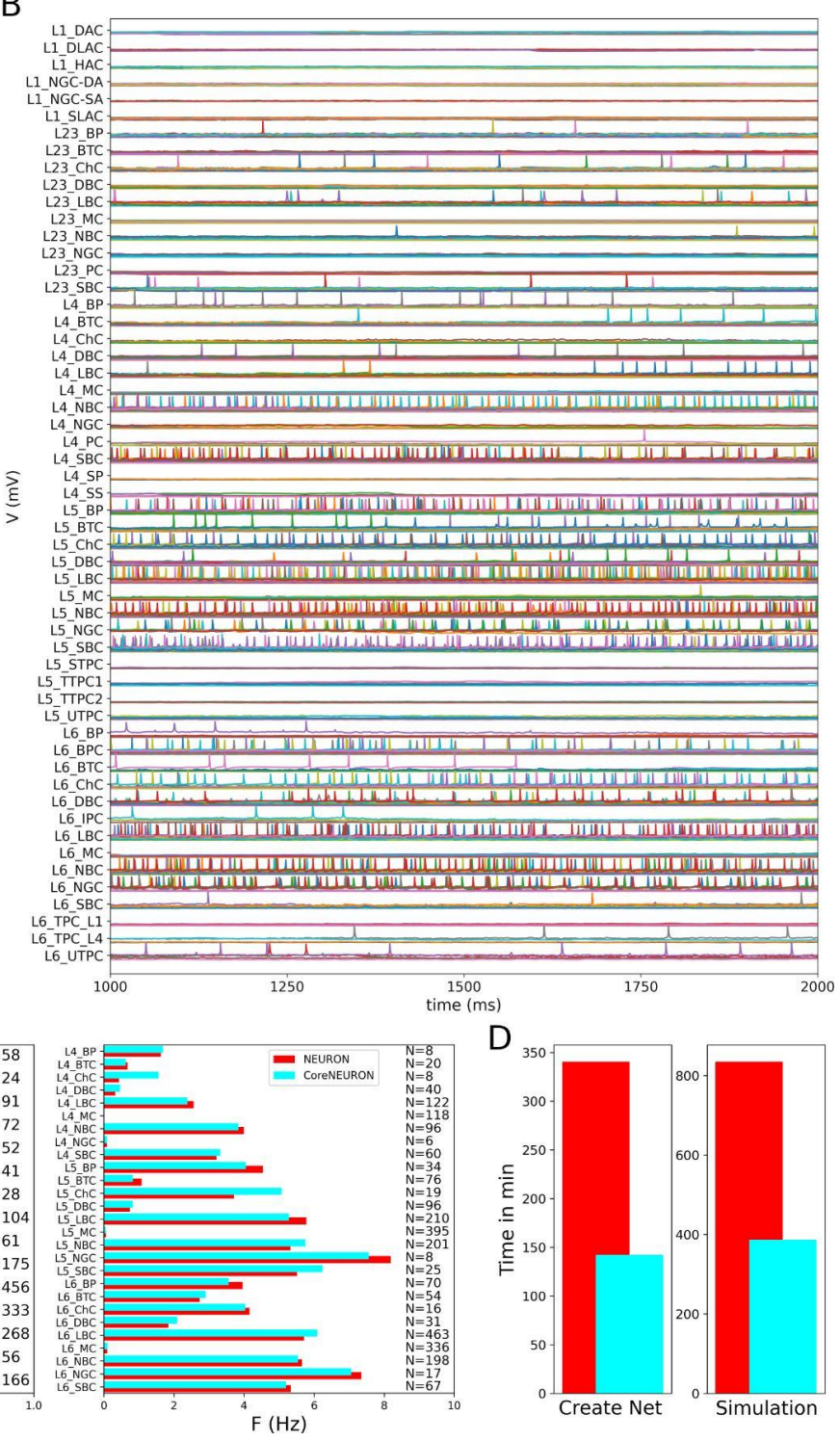

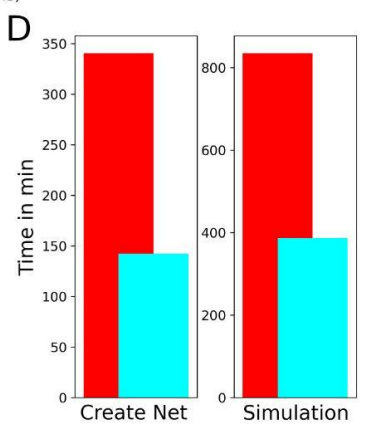

Figure 5. NetPyNE S1 circuit response to background inputs (spontaneous activity). (A) Spiking raster plot for all the 31,346 cells in the S1 column during 1 second. (B) Example voltage traces for each of the 207 me-types grouped by rows into their respective $55 \mathrm{~m}$-types. (C) Mean firing rates of each of the $55 \mathrm{~m}$-types for NEURON (red) vs CoreNEURON (cyan). (D) Comparison of the time required to create the network and run the simulation on a 40-core Google Cloud virtual machine using NEURON (red) or CoreNEURON (cyan). 
bioRxiv preprint doi: https://doi.org/10.1101/2022.02.03.479029; this version posted February 6, 2022. The copyright holder for this preprint (which was not certified by peer review) is the author/funder. All rights reserved. No reuse allowed without permission.

Large-scale model of somatosensory thalamocortical circuits

\subsection{Full cortico-thalamo-cortical circuit response with with short term plasticity}

First, we simulated the response of the cortical and thalamic circuits independently (not interconnected) but including short term plasticity (STP) in the S1 recurrent connections (Fig. 6A). STP resulted in emergence of synchronous bursting within the S1 cortical column at approximately 1 $\mathrm{Hz}$ frequency (compare S1 raster in Figs. 5A and 6A). The spontaneous synchronous bursts first appeared in L5, and then spread to all S1 cells within $100 \mathrm{~ms}$. Fig. 6C shows an amplified raster plot of L4-L6 with $70 \mathrm{~ms}$ of activity at the time when spontaneous synchronous bursts started.

The thalamic populations (disconnected from S1 and without STP) also exhibited stable self-sustained activity with rhythmic bursting at theta $(\sim 6 \mathrm{~Hz})$, although oscillations were most clear in the VPL and POm populations (Fig 6A, cells 31,346 to 38,612).

We then simulated the full circuit with bidirectional connections between S1 and thalamus and STP in the thalamus to S1 connections (Fig. 6B). The full cortico-thalamo-cortical circuit exhibited a higher mean firing rate for most S1 populations, and spontaneous synchronous bursts were more frequent in S1 and stronger in thalamus, and were now synchronized across all S1 and thalamic populations. Fig. 6D shows the voltage traces of several cortical and thalamic neurons, illustrating the spike synchrony of S1 and thalamic populations.
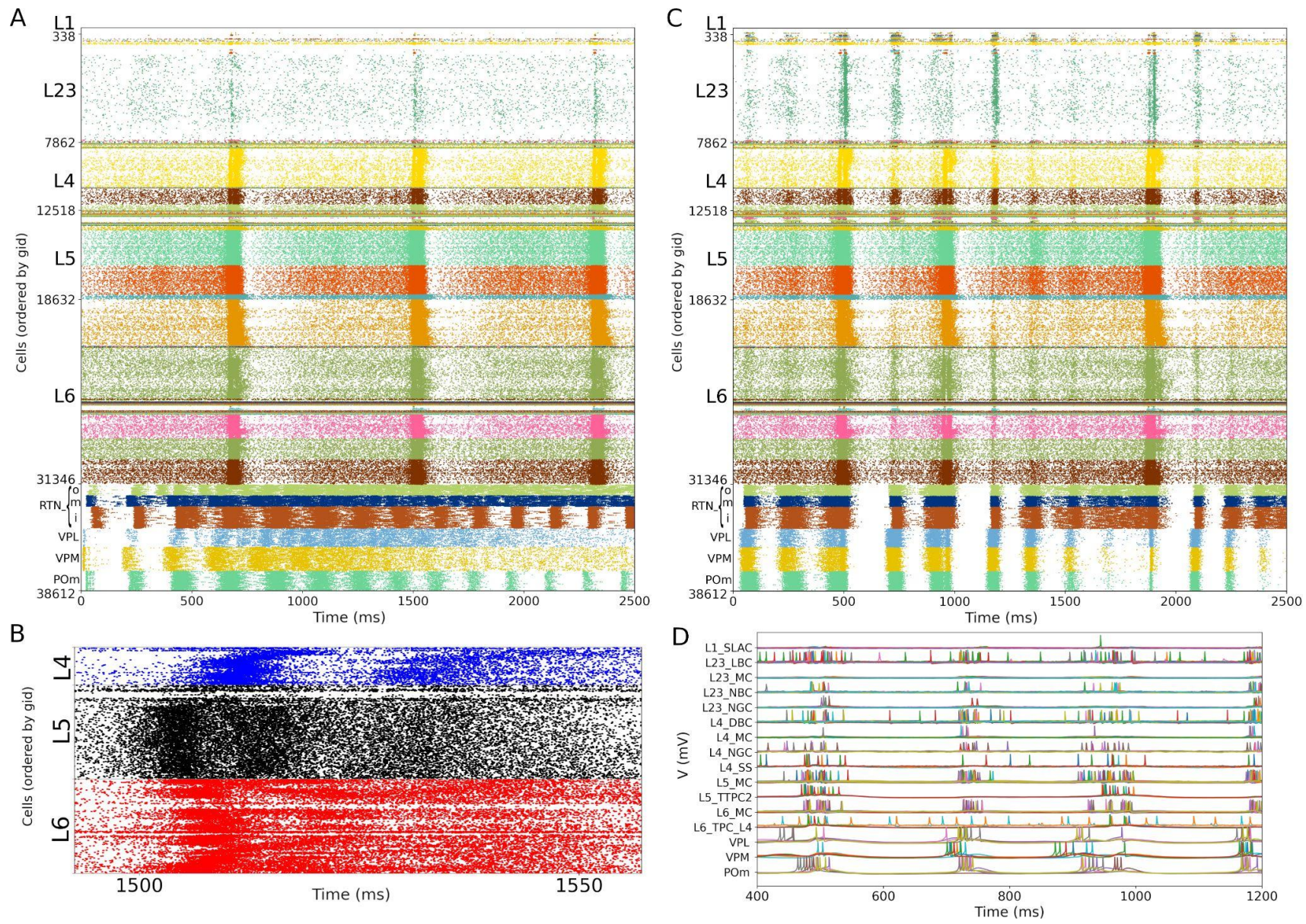

Figure 6. NetPyNE S1 and thalamic circuit response with short-term plasticity (STP). (A) Spiking raster plot of S1 with STP and thalamic populations (in order from top: RTN_o, RTN_m, RTN_i, VPL, VPM, and POm). S1 and thalamus are not interconnected; only intracortical and intrathalamic connections are included. (B) Amplified spiking raster plot (A) showing the $70 \mathrm{~ms}$ around the time when synchronous bursts first occur 
bioRxiv preprint doi: https://doi.org/10.1101/2022.02.03.479029; this version posted February 6, 2022. The copyright holder for this preprint

(which was not certified by peer review) is the author/funder. All rights reserved. No reuse allowed without permission.

Large-scale model of somatosensory thalamocortical circuits

in L5 (black) and then propagate to L6 (red) and L4 (blue). (C) Spiking raster plot of the fully connected circuit model, including bidirectional connections between $\mathrm{S} 1$ and thalamus. Oscillations at $\sim 6 \mathrm{~Hz}$ were now synchronized across all S1 and thalamic populations. (D) Example traces from (C) during $800 \mathrm{~ms}$ showing spike synchrony across cortical and thalamic populations. Rasters in A and C show 2.5 seconds after steady state was reached.

\section{Discussion}

We provided here the first large-scale S1 model that is accessible to the wider community, building on the details of the prior state-of-the-art BBP S1 model. The model closely reproduced the original cell morphologies and electrophysiological responses for the 207 morpholectric (me) cell types, with 5 examples for each, totaling 1035 cell models (Fig. 1); the spatial distribution of these cells across layers; and the connectivity properties of the 1941 pathways, including synaptic dynamics and short-term plasticity (Figs. 2,3). After tuning, the simulations produced reasonable dynamics with rates and activity patterns corresponding to in vivo measures of cortical activity (Figs. 5,6). There was no direct comparison to the full network dynamics of the original BBP model since original simulation data was not available. However, firing rates and overall $1 \mathrm{~Hz}$ underlying oscillation when using STP is comparable to that seen in the original model version paper (Fig 11). We also extended the model by adding thalamic circuits, including 6 distinct thalamic populations with intrathalamic, thalamocortical and corticothalamic connectivity derived from experimental data (Fig. 4). The addition of the thalamic circuit resulted in distinct activity patterns and synchronous activity across cortical and thalamic populations (Figs. 6B).

The S1 model now joins other NetPyNE cortical simulations: generic cortical circuits (Romaro et al. 2021), auditory and motor thalamocortical circuits (Dura-Bernal et al., n.d.; Sivagnanam et al. 2020), as well as simulations of thalamus (Moreira et al. 2021), dorsal horn of spinal cord (Sekiguchi et al. 2021), Parkinson's disease (Ranieri et al. 2021) and schizophrenia (Metzner et al. 2020). These large cortical simulations can be extremely computer-intensive, which is a major motivation for NetPyNE's facilities that allow one to readily simplify the network by swapping in integrate-and-fire or small-compartmental cell models, or by down-scaling to more manageable sizes. The optimization on CPUs and the ability to run across GPUs in CoreNEURON is another key NetPyNE feature enhancing runnability. In the present case, the original S1 model is largely inaccessible, despite the cooperation of its designers, since it requires specialized tools, workflows, and training. Nonetheless, most of the data required to replicate it is available via the NMCP, which we ourselves used to implement the NetPyNE version.

We made 2 significant changes in our port to NetPyNE. First, we did not replicate the stochastic K channels that appear in $3.6 \%$ of the neurons, making our port somewhat simpler than the original. This channel required writing custom code and made simulations slower, but it could be added to the model in a future iteration. Second, we have not utilized the original cell-to-cell connection mappings that were obtained by BBP from direct microscopic observations of overlap between pre-synaptic axonal fields and post-synaptic dendritic fields (so-called Peter's principle). In the original BBP S1 model, the use of cell-to-cell connections necessarily limited the simulation to use precisely the original models cell morphologies, cell positions and scales. It also required storing and loading large files of connection data. We therefore replaced this connection framework with one based on connection probability based on cell type (including layer), inter-cell distance, and dendritic pattern of post-synaptic locations. Although saving somewhat on space, there is a time-space tradeoff since 
bioRxiv preprint doi: https://doi.org/10.1101/2022.02.03.479029; this version posted February 6, 2022. The copyright holder for this preprint

(which was not certified by peer review) is the author/funder. All rights reserved. No reuse allowed without permission.

Large-scale model of somatosensory thalamocortical circuits

this requires further calculations on start-up. Despite these limitations, we had excellent agreement with both cell model matching and connection density matching.

We were able to get substantial speedup $(>2 \mathrm{x})$ for both model setup and run using CoreNEURON despite only using CPUs with no GPU at this time. The differences in firing seen with NEURON vs CoreNEURON are expected due to vectorisation of the compute kernels in CoreNEURON and potential differences due to different solvers when using NMODL with sympy. Further differences are to be expected once this is extended to GPUs (Kumbhar et al. 2019; Jézéquel, Lamotte, and Saïd 2015).

Our new port of the S1 model provides a quantitative framework that can be used in several ways. First, it can be used to perform in silico experiments to explore somatosensory processing under the assumption of various coding paradigms or brain disease (Amsalem et al., n.d.; Metzner et al. 2020; Ranieri et al. 2021). Second, drug effects can be directly tested in the simulation (Neymotin et al. 2016) -- this is an advantage of a multiscale model with scales from molecule to network, which is not available in simpler models that elide these details. Third, the model constitutes a unified multiscale framework for organizing our knowledge of S1 which serves as a dynamical database to which new physiological, transcriptomic, proteomic, and anatomical data can be added. This framework can then be utilized as a community tool for researchers in the field to test hypotheses and guide the design of new experiments.

\section{Acknowledgments}

This work was funded by the following grants: NIH U24EB028998, NSF 1904444-1042C and NYS SCIRB DOH01-C32250GG-3450000.

\section{$6 \quad$ References}

Amsalem, Oren, James King, Michael Reimann, Srikanth Ramaswamy, Eilif Muller, Henry Markram, Israel Nelken, and Idan Segev. n.d. "Dense Computer Replica of Cortical Microcircuits Unravels Cellular Underpinnings of Auditory Surprise Response.” BioRxiv. https://doi.org/10.1101/2020.05.31.126466.

Azarfar, Alireza, Niccoló Calcini, Chao Huang, Fleur Zeldenrust, and Tansu Celikel. 2018. "Neural Coding: A Single Neuron's Perspective." Neuroscience and Biobehavioral Reviews 94 (November): 238-47.

Barthas, Florent, and Alex C. Kwan. 2017. "Secondary Motor Cortex: Where 'Sensory' Meets 'Motor' in the Rodent Frontal Cortex." Trends in Neurosciences 40 (3): 181-93.

Carnevale, Ted, and Michael Hines. 2006. The NEURON Book. New York: Cambridge University Press.

Dai, Kael, Juan Hernando, Yazan N. Billeh, Sergey L. Gratiy, Judit Planas, Andrew P. Davison, Salvador

Dura-Bernal, et al. 2020. "The SONATA Data Format for Efficient Description of Large-Scale Network Models.” PLoS Computational Biology 16 (2): e1007696.

Destexhe, A., T. Bal, D. A. McCormick, and T. J. Sejnowski. 1996. "Ionic Mechanisms Underlying Synchronized Oscillations and Propagating Waves in a Model of Ferret Thalamic Slices." Journal of Neurophysiology 76 (3): 2049-70.

Dura-Bernal, Salvador, Samuel A. Neymotin, Benjamin A. Suter, Gordon M. G. Shepherd, and William W. Lytton. n.d. "Multiscale Dynamics and Information Flow in a Data-Driven Model of the Primary Motor Cortex Microcircuit." https://doi.org/10.1101/201707.

Dura-Bernal, Salvador, Benjamin A. Suter, Padraig Gleeson, Matteo Cantarelli, Adrian Quintana, Facundo Rodriguez, David J. Kedziora, et al. 2019. "NetPyNE, a Tool for Data-Driven Multiscale Modeling of Brain Circuits.” eLife 8 (April). https://doi.org/10.7554/eLife.44494. 
bioRxiv preprint doi: https://doi.org/10.1101/2022.02.03.479029; this version posted February 6, 2022. The copyright holder for this preprint (which was not certified by peer review) is the author/funder. All rights reserved. No reuse allowed without permission.

Large-scale model of somatosensory thalamocortical circuits

Erö, Csaba, Marc-Oliver Gewaltig, Daniel Keller, and Henry Markram. 2018. "A Cell Atlas for the Mouse Brain." Frontiers in Neuroinformatics 12 (November): 84.

Fuhrmann, Galit, Idan Segev, Henry Markram, and Misha Tsodyks. 2002. "Coding of Temporal Information by Activity-Dependent Synapses.” Journal of Neurophysiology 87 (1): 140-48.

Gal, Eyal, Michael London, Amir Globerson, Srikanth Ramaswamy, Michael W. Reimann, Eilif Muller, Henry Markram, and Idan Segev. 2017. "Rich Cell-Type-Specific Network Topology in Neocortical Microcircuitry." Nature Neuroscience 20 (7): 1004-13.

Gleeson, Padraig, Sharon Crook, Robert C. Cannon, Michael L. Hines, Guy O. Billings, Matteo Farinella, Thomas M. Morse, et al. 2010. "NeuroML: A Language for Describing Data Driven Models of Neurons and Networks with a High Degree of Biological Detail." PLoS Computational Biology 6 (6): e1000815.

Guo, Kuanghua, Naoki Yamawaki, John M. Barrett, Martinna Tapies, and Gordon M. G. Shepherd. 2020. "Cortico-Thalamo-Cortical Circuits of Mouse Forelimb S1 Are Organized Primarily as Recurrent Loops." The Journal of Neuroscience: The Official Journal of the Society for Neuroscience 40 (14): 2849-58.

Hagen, Espen, Solveig Næss, Torbjørn V. Ness, and Gaute T. Einevoll. 2018. "Multimodal Modeling of Neural Network Activity: Computing LFP, ECoG, EEG, and MEG Signals With LFPy 2.0.” Frontiers in Neuroinformatics. https://doi.org/10.3389/fninf.2018.00092.

Hennig, Matthias H. 2013. "Theoretical Models of Synaptic Short Term Plasticity.” Frontiers in Computational Neuroscience 7 (April): 45.

Hill, Sean, and Giulio Tononi. 2005. "Modeling Sleep and Wakefulness in the Thalamocortical System." Journal of Neurophysiology 93 (3): 1671-98.

Iavarone, Elisabetta, Jane Yi, Ying Shi, Bas-Jan Zandt, Christian O’Reilly, Werner Van Geit, Christian Rössert, Henry Markram, and Sean L. Hill. 2019. "Experimentally-Constrained Biophysical Models of Tonic and Burst Firing Modes in Thalamocortical Neurons." PLoS Computational Biology 15 (5): e1006753.

Izhikevich, Eugene M., and Gerald M. Edelman. 2008. "Large-Scale Model of Mammalian Thalamocortical Systems." Proceedings of the National Academy of Sciences of the United States of America 105 (9): 3593-98.

Jézéquel, Fabienne, Jean-Luc Lamotte, and Issam Saïd. 2015. "Estimation of Numerical Reproducibility on CPU and GPU." In Proceedings of the 2015 Federated Conference on Computer Science and Information Systems. IEEE. https://doi.org/10.15439/2015f29.

Kumbhar, Pramod, Michael Hines, Jeremy Fouriaux, Aleksandr Ovcharenko, James King, Fabien Delalondre, and Felix Schürmann. 2019. "CoreNEURON : An Optimized Compute Engine for the NEURON Simulator." Frontiers in Neuroinformatics 13 (September): 63.

Lam, Ying-Wan, Christopher S. Nelson, and S. Murray Sherman. 2006. "Mapping of the Functional Interconnections Between Thalamic Reticular Neurons Using Photostimulation." Journal of Neurophysiology 96 (5): 2593-2600.

Lam, Ying-Wan, and S. Murray Sherman. 2007. "Different Topography of the Reticulothalmic Inputs to Firstand Higher-Order Somatosensory Thalamic Relays Revealed Using Photostimulation." Journal of Neurophysiology 98 (5): 2903-9.

- 2011. "Functional Organization of the Thalamic Input to the Thalamic Reticular Nucleus." The Journal of Neuroscience: The Official Journal of the Society for Neuroscience 31 (18): 6791-99.

Łuczyńska, Anna, Jerzy Dziewiątkowski, Hanna Jagalska-Majewska, Przemysław Kowiański, Sławomir Wójcik, Cezary Labuda, and Janusz Moryś. 2003. "Qualitative and Quantitative Analysis of the Postnatal Development of the Ventroposterolateral Nucleus of the Thalamus in Rat and Rabbits." Folia Mophologica 62 (2): 75-87.

Lytton, William W., Alexandra Seidenstein, Salvador Dura-Bernal, Felix Schurmann, Robert A. McDougal, and Michael L. Hines. 2016. "Simulation Neurotechnologies for Advancing Brain Research: Parallelizing Large Networks in NEURON." Neural Computation 28: 2063-90.

Ma, P. M. 1991. "The Barrelettes--Architectonic Vibrissal Representations in the Brainstem Trigeminal Complex of the Mouse. I. Normal Structural Organization." The Journal of Comparative Neurology 309 (2): 161-99.

Markram, Henry, Eilif Muller, Srikanth Ramaswamy, Michael W. Reimann, Marwan Abdellah, Carlos Aguado Sanchez, Anastasia Ailamaki, et al. 2015. "Reconstruction and Simulation of Neocortical Microcircuitry." Cell 163 (2): 456-92. 
bioRxiv preprint doi: https://doi.org/10.1101/2022.02.03.479029; this version posted February 6, 2022. The copyright holder for this preprint (which was not certified by peer review) is the author/funder. All rights reserved. No reuse allowed without permission.

Large-scale model of somatosensory thalamocortical circuits

McDougal, R., A. Bulanova, and W. Lytton. 2016. "Reproducibility in Computational Neuroscience Models and Simulations." IEEE Transactions on Biomedical Engineering.

Metzner, Christoph, Tuomo Mäki-Marttunen, Gili Karni, Hana McMahon-Cole, and Volker Steuber. 2020. "The Effect of Alterations of Schizophrenia-Associated Genes on Gamma Band Oscillations." bioRxiv. bioRxiv. https://doi.org/10.1101/2020.09.28.316737.

Meyer, Hanno S., Verena C. Wimmer, Mike Hemberger, Randy M. Bruno, Christiaan P. J. de Kock, Andreas Frick, Bert Sakmann, and Moritz Helmstaedter. 2010. "Cell Type-Specific Thalamic Innervation in a Column of Rat Vibrissal Cortex." Cerebral Cortex 20 (10): 2287-2303.

Migliore, Michele, C. Cannia, William W. Lytton, Henry Markram, and Michael L. Hines. 2006. "Parallel Network Simulations with NEURON." Journal of Computational Neuroscience 21 (2): 119-29.

Moreira, Joao V. S., Fernando S. Borges, Donald Doherty, William W. Lytton, and Salvador Dura-Bernal. 2021. "Topographically Detailed Computational Model of the Motor and Somatosensory Thalamic Circuits." Presented at the Neuroscience 2021, Online. https://www.abstractsonline.com/pp8/\#!/10485/presentation/16321.

Murray, John D., and Alan Anticevic. 2017. "Toward Understanding Thalamocortical Dysfunction in Schizophrenia through Computational Models of Neural Circuit Dynamics." Schizophrenia Research 180 (February): 70-77.

Neymotin, Samuel A., Salvador Dura-Bernal, Herman Moreno, and William W. Lytton. 2016. "Computer Modeling for Pharmacological Treatments for Dystonia." Drug Discovery Today. Disease Models 19: 51-57.

Ohno, Sachi, Eriko Kuramoto, Takahiro Furuta, Hiroyuki Hioki, Yasuhiro R. Tanaka, Fumino Fujiyama, Takahiro Sonomura, Masanori Uemura, Kazuna Sugiyama, and Takeshi Kaneko. 2012. "A Morphological Analysis of Thalamocortical Axon Fibers of Rat Posterior Thalamic Nuclei: A Single Neuron Tracing Study with Viral Vectors." Cerebral Cortex 22 (12): 2840-57.

O’Reilly, Christian, Elisabetta Iavarone, Jane Yi, and Sean L. Hill. 2021. "Rodent Somatosensory Thalamocortical Circuitry: Neurons, Synapses, and Connectivity." Neuroscience and Biobehavioral Reviews 126 (July): 213-35.

Peña-Rangel, Teresa M., Paola Isabel Lugo-Picos, Ana Silvia Báez-Cordero, Ana Elizabeth Hidalgo-Balbuena, Annie Yolene Luma, Ana Karen Pimentel-Farfan, and Pavel E. Rueda-Orozco. 2021. "Altered Sensory Representations in Parkinsonian Cortical and Basal Ganglia Networks." Neuroscience 466 (July): 10-25.

Petrof, Iraklis, Angela N. Viaene, and S. Murray Sherman. 2015. "Properties of the Primary Somatosensory Cortex Projection to the Primary Motor Cortex in the Mouse." Journal of Neurophysiology 113 (7): 2400-2407.

Potjans, Tobias C., and Markus Diesmann. 2014. "The Cell-Type Specific Cortical Microcircuit: Relating Structure and Activity in a Full-Scale Spiking Network Model." Cerebral Cortex 24 (3): 785-806.

Ramaswamy, Srikanth, Jean-Denis Courcol, Marwan Abdellah, Stanislaw R. Adaszewski, Nicolas Antille, Selim Arsever, Guy Atenekeng, et al. 2015. "The Neocortical Microcircuit Collaboration Portal: A Resource for Rat Somatosensory Cortex." Frontiers in Neural Circuits 9 (October): 44.

Ranieri, Caetano M., Jhielson M. Pimentel, Marcelo R. Romano, Leonardo A. Elias, Roseli A. F. Romero, Michael A. Lones, Mariana F. P. Araujo, Patricia A. Vargas, and Renan C. Moioli. 2021. "A Data-Driven Biophysical Computational Model of Parkinson's Disease Based on Marmoset Monkeys." IEEE Access: Practical Innovations, Open Solutions 9: 122548-67.

Reimann, Michael W., Anna-Lena Horlemann, Srikanth Ramaswamy, Eilif B. Muller, and Henry Markram. 2017. "Morphological Diversity Strongly Constrains Synaptic Connectivity and Plasticity." Cerebral Cortex 27 (9): 4570-85.

Reimann, Michael W., James G. King, Eilif B. Muller, Srikanth Ramaswamy, and Henry Markram. 2015. “An Algorithm to Predict the Connectome of Neural Microcircuits." Frontiers in Computational Neuroscience 9 (October): 120.

Reimann, Michael W., Max Nolte, Martina Scolamiero, Katharine Turner, Rodrigo Perin, Giuseppe Chindemi, Paweł Dłotko, Ran Levi, Kathryn Hess, and Henry Markram. 2017. "Cliques of Neurons Bound into Cavities Provide a Missing Link between Structure and Function." Frontiers in Computational Neuroscience 11: 48.

Reimann, M. W., E. B. Muller, S. Ramaswamy, and H. Markram. 2015. “An Algorithm to Predict the 
bioRxiv preprint doi: https://doi.org/10.1101/2022.02.03.479029; this version posted February 6, 2022. The copyright holder for this preprint (which was not certified by peer review) is the author/funder. All rights reserved. No reuse allowed without permission.

Large-scale model of somatosensory thalamocortical circuits

Connectome of Neural Microcircuits', Frontiers in Computational Neuroscience." Frontiers 9. $\mathrm{https}$ ://www.scopus.com/record/display.uri?eid=2-s2.0-84943656352\&origin=inward\&featureToggles=F EATURE_VIEW_PDF:1.

Romaro, Cecilia, Fernando Araujo Najman, William W. Lytton, Antonio C. Roque, and Salvador Dura-Bernal. 2021. "NetPyNE Implementation and Rescaling of the Potjans-Diesmann Cortical Microcircuit Model." Neural Computation.

Sekiguchi, Kazutaka, Laura Medlock, Salvador Dura-Bernal, Steven A. Prescott, and William W. Lytton. 2021. "Multiscale Computer Model of the Spinal Dorsal Horn Reveals Changes in Network Processing Associated with Chronic Pain." bioRxiv. https://doi.org/10.1101/2021.06.09.447785.

Shepherd, Gordon M. G., and Naoki Yamawaki. 2021. "Untangling the Cortico-Thalamo-Cortical Loop: Cellular Pieces of a Knotty Circuit Puzzle.” Nature Reviews. Neuroscience 22 (7): 389-406.

Sivagnanam, Subhashini, Wyatt Gorman, Donald Doherty, Samuel A. Neymotin, Stephan Fang, Hermine Hovhannisyan, William W. Lytton, and Salvador Dura-Bernal. 2020. "Simulating Large-Scale Models of Brain Neuronal Circuits Using Google Cloud Platform." PEARC20 : Practice and Experience in Advanced Research Computing 2020 : Catch the Wave : July 27-31, 2020, Portland, Or Virtual Conference. Practice and Experience in Advanced Research Computing (Conference) (2020 : Online) 2020 (July): 505-9.

Sugitani, M., J. Yano, T. Sugai, and H. Ooyama. 1990. "Somatotopic Organization and Columnar Structure of Vibrissae Representation in the Rat Ventrobasal Complex." Experimental Brain Research. Experimentelle Hirnforschung. Experimentation Cerebrale 81 (2): 346-52.

Vázquez, Yuriria, Emilio Salinas, and Ranulfo Romo. 2013. "Transformation of the Neural Code for Tactile Detection from Thalamus to Cortex." Proceedings of the National Academy of Sciences of the United States of America 110 (28): E2635-44.

\section{Data Availability Statement}

The datasets for this study can be found in the github.com/suny-downstate-medical-center/S1_netpyne/. 